\title{
Los Museos y las Tecnologías de la Comunicación en Cuenca
}

\section{Museums and Communication Technologies in Cuenca}

\author{
Brígida Ximena San Martín García ${ }^{1 *}$ y Édgar Vicente Cordero Coellar ${ }^{2}$ \\ ${ }^{1}$ Diario El Mercurio \\ ${ }^{2}$ Universidad Católica de Cuenca \\ *rferfi@yahoo.fr
}

DOI: https://doi.org/10.26871/killkana_social.v2i3.346

\begin{abstract}
Resumen
Las tecnologías y la Internet han cambiado el modelo de acción dentro del ámbito museístico. Museos Interconectados, museos interactivos, las tecnologías de la información y comunicación, transforma el contacto con los públicos. Algunos museos y centros culturales de Cuenca incorporan nuevas estrategias de comunicación virtual apoyadas en plataformas de la web 2.0 y con aplicaciones que permitan una mayor interactividad e interconexión. Estos museos y Centros Culturales apuestan a estas plataformas por el mayor nivel de acceso a los públicos e interacción con centros culturales de cualquier parte del planeta. Investigar los procesos de divulgación de discursos informativos de estos espacios, requiere de una relación directa con la actividad de cada uno de ellos, convertirse en seguidor de sus cuentas de redes sociales y mantener diálogos con directivos, actores y gestores culturales que dan vida a estos espacios.
\end{abstract}

Palabras clave: museos, centros culturales, divulgación, información, plataformas digitales, aplicaciones, web, investigación, Cuenca, Ecuador.

\begin{abstract}
The Technologies and the internet have changed the model of action within the museum field. Interconnected museums, interactive museums, information and communication technologies, Tics, transforms contact with the public. Some museums and cultural centers of the Basin incorporate new strategies of virtual communication supported by web 2.0 platforms and with applications that allow greater interactivity and interconnection. The Museums and Cultural Centers bet on these platforms for the highest level of access to public and interaction with cultural centers anywhere in the world. Investigate the processes of dissemination of information discourses of these spaces, requires a direct relationship with the activity of each of them, become a follower of their social network accounts and maintain dialogues with managers, actors and cultural managers that give life to these spaces.
\end{abstract}

Key words: museums, cultural centers, dissemination, information, digital platforms, applications, web, research, Cuenca, Ecuador.

\section{Introducción}

La característica de los museos de trabajar el "para siempre" no cambia, a pesar de las transformaciones sociales, políticas, económicas, dictadas por las tecnologías de la comunicación y todo el embrollo de la Sociedad de la Información. Ahora, en el siglo XXI, la cuestión no es trabajar con la mirada a ese "para siempre", sino todo un siempre.

Los museos y centros culturales públicos o privados son portadores de cultura, pero también son agentes de cambio social (Acha Barral, 2006) y en ese sentido el conocimiento que allí se genera trasciende en el espacio donde se ubica y a donde se orienta.

El presente estudio tomo como eje de análisis de los contenidos de la información que circula a través de las páginas de redes sociales de algunos museos y espacios culturales independientes de la ciudad. El objetivo de este acercamiento virtual y físico a estos centros permite adentrarse -en parte- a la presencia de la acción museística, artística y cultural en las comunidades virtuales y enrolarse con las fórmulas que aplican para la generación de información expuesta en sus plataformas, quienes son los receptores, y, los niveles de interacción con los mismos.

La investigación de esta naturaleza no puede dejar de lado aquel principio anotado por Manuel Catells (Castells, 1997) sobre las nuevas tecnologías de la información que integran al mundo en redes globales de instrumentalidad, a esa comunicación que se da a través del ordenador y engendra un vasto despliegue de comunidades virtuales, al paradigma del informacionalismo, en el que los cambios tecnológicos impactan en las nuevas estructuras sociales, 
por un lado y en las fuerzas económicas productivas, por otro.

Cuentas de Facebook, Twitter, Instagram, YouTube con miles de seguidores y actualizaciones que causan vértigo porque parecen más veloces que el mismo tiempo; páginas web que se han dormido en una multimedialidad vaga, dan cuenta de la percepción que la gestión cultural de hoy tiene con relación a la divulgación de la información y a las relaciones con la sociedad.

Este trabajo apunta a investigar para generar el debate y el análisis comunicacional e informacional de los discursos que los espacios culturales difunden en las redes y a través de aplicaciones móviles.

\section{Marco Teorico}

En los museos y en los espacios culturales independientes las tecnologías ganan terreno. En un principio, las herramientas tecnológicas no sólo referían a las TICs, sino a cómo en los años 80 y 90 se instalaron los primeros ordenadores que permitieron las primeras interacciones. El proceso de archivar información en ordenadores abrió otra posibilidad, de guardar y poner en circulación dicha información a través de soportes como del DVD, el Disco Compacto, memorias portátiles.

El mundo de la informática y el gran salto tecnológico con la llegada de la Internet se convirtieron en las herramientas número uno para la materialización de los requerimientos necesarios, desaparecer la obligatoriedad de la visita física para impregnarse del conocimiento allí contenido, así como las restricciones horarias, pues el museo permanece abierto las 24 horas del día, todos los días del año. (Martínez-Sanz, 2012)

Está claro que las tecnologías y la Internet, que abrieron paso a la web 2.0, han cambiado el modelo de acción dentro del ámbito museístico Museos Interconectados, museos interactivos, las Tecnologías de la Información y Comunicación, Tics, transforman el contacto con los públicos. La comunicación y enlaces que ofrece esta comunicación medida por computadora alteró esa relación museo públicos y del público a los museos, ahora las dimensiones de interactividad son mayores, provocados por la hiperconectividad que ofrecen las redes sociales, las páginas web y los blogs.

Los museos incorporan de a poco y de manera más vertiginosa nuevas estrategias virtuales apoyadas en plataformas de la web 2.0, como son las herramientas para ordenadores y dispositivos móviles que permiten un acceso más fácil para el público, esto provoca cierta participación más activa hasta ofrecer una experiencia complementaria a la visita física.

Ningún museo escapa de la revuelta tecnológica y no solo en materia de equipamientos técnicos intramuseo. Ahora ponen énfasis en el manejo de información que publican en las pantallas tecnológicas táctiles, la geolocalización, la realidad aumentada, los códigos QR, los podcasts, las redes sociales, la digitalización de documentos, el crowdfunding, consiguiendo que el museo traspase sus muros, convirtiéndolo en un lugar más social y dinámico.

En ciudades donde la acción museística es un componente importante de la producción cultural, la conexión de los museos es indispensable no solo para la divulgación de información, sino para la interrelación con diversos actores: a) los públicos que de por sí son múltiples; b) otros museos que en conjunto estructuran comunidades y redes; c) actores culturales que son quienes mueven en parte la producción de la programación; d) artistas, críticos, antropólogos, que son la base del desarrollo del pensamiento.

Las posibilidades de comunicación y de difusión de las actividades e imagen del museo dada por el uso de las plataformas digitales se multiplican y con ella la participación del público para la interacción y la construcción de contenidos, en este sentido, la web 2.0 da la posibilidad al público además de la participación directa, generar información y ser parte del proceso informativo. (Fiallos Quinteros, 2015)

La evolución de las plataformas de la información, no sólo revolucionaron la comunicación, sino todas las actividades humanas, y por ende el mundo del arte. Hoy en día no hay propuesta de obra o práctica artística que no aproveche los nuevos canales tecnológicos como soportes o como canales para llegar a los públicos.

Felisita Casillo, en el libro Estudios de Arte y Comunicación de José Luis Crespo (Crespo, 2012) califica a la Internet como una amable galería virtual. Para ella, entre las enormes ventajas que posibilitó Internet están: la comunicación simultánea, la comercialización directa, la búsqueda de información de cualquier tipo, y, por cierto, la difusión del arte que, en su criterio, ha sido una de las actividades más beneficiadas.

Con la incidencia de la tecnología en la difusión de información, las plataformas de comunicación como los blogs, páginas web, cuentas de redes sociales, son las que inciden en la forma de informar. Como dice José Luis Crespo: ahora, Internet es el objeto de las más recientes investigaciones. Cada vez más nos adentramos en una cyber-sociedad dependiente de las inmensas oportunidades de comunicación que las nuevas tecnologías proporcionan.

Este fenómeno se acrecienta con la aparición de las redes, espacios por los cuales, los actores culturales optaron por dirigirse a los públicos que, sin duda, son diversos a los de los medios tradicionales y masivos, abriendo entonces una brecha y a la vez una relación informativa entre el periodismo para medios convencionales y el periodismo para soportes digitales.

Las artes como materia de información se mueven a través de las plataformas y de las concepciones informativas que ellas despiertan; es decir la difusión e intercambio de información, posibilitan que, el Periodismo de Cultura salte a las nuevas plataformas y gane públicos.

\section{Metodología}

La investigación tiene como objetivo conocer cómo se realiza el proceso de divulgación de la información que 
generan algunos museos y centros culturales independientes de la ciudad; por lo tanto el proceso investigativo se basa en el análisis a la acción comunicativa que, a través de la red Facebook y las páginas web despliegan algunos centros culturales y museos de la ciudad; como también la utilización de aplicaciones móviles disponibles para el acercamiento virtual a los espacios expositivos y muestras permanentes que mantienen estos espacios. Estudiar las formas y características de estos procesos informativos con la revisión de las plataformas digitales y la observación in situ de estos lugares y la utilización de las herramientas por ellos aplicadas para los públicos, es la metodología empleada para esta investigación

Este proyecto de investigación tiene la modalidad descriptiva. Estos tipos de investigación suelen ser las etapas cronológicas de todo estudio científico y cada una tiene una finalidad diferente: primero se 'explora' la difusión de información de productos culturales en soportes digitales. 'Describe' las variables involucradas, que son los medios digitales como canales en la difusión de productos culturales de los museos y centros culturales de la ciudad.

Luego de ello se 'correlacionan' las variables entre sí, la variable independiente que son los medios digitales, con la dependiente que son los procesos de difusión; de esa manera se obtuvieron resultados para tratar de explicar la influencia de las variables independientes sobre las dependientes.

\section{Resultados}

\subsection{Museos de Cuenca le apuestan a la red}

\subsubsection{El Museo de Arte Moderno}

En Cuenca, en el Museo de Arte Moderno, MMAM, el uso de las tecnologías no solo engloba a la instalación de equipos dentro del espacio museo. La acción tiene la visión de lograr mayor alcance comunicativo y participativo con el ingreso a las redes sociales, manteniendo cuentas en redes de Facebook, en las cuales sus seguidores llegan a 5000; como también en la cuenta de Instagram, donde registra 827, sumando a eso la cuenta de twitter.

Información de las exposiciones, horarios, promoción de actividades orientadas a niños y a la acción con el entorno físico de museo están en el muro. Fotos, videos, collages, texto con hipervínculos, galerías, líneas de tiempo, son algunas de las formas de comunicar a través de estas plataformas digitales

La retroalimentación, los enlaces y contactos fuera de las geografías locales, es lo que sorprende a quienes manejan la información y comunicación mediada por la computadora, cada día a día los seguidores en Facebook y en las otras cuentas aumentan.

Este es un fenómeno universal como la internet misma. En la fase final del 2017, el Museo de El Prado de España, puso en marcha nuevos proyectos educativos basados en la tecnología. En acuerdo con el gigante Samsung, y a través del empleo de tabletas se propuso una experiencia multimedia e interactiva relacionada con las colecciones.
La elaboración de contenidos culturales de un museo se materializa en diferentes soportes de mediación desde los tradicionales, como las exposiciones, textos de sala, escenografías, maquetas, catálogos y guías de visita, audioguías, audiovisuales, etc., a otros más recientes, como las recreaciones virtuales, guías multimedia, aplicaciones móviles, etc. Y si se habla de comunicación cultural se habla de ideas. No se comunican obras de arte, documentos o restos arqueológicos, se comunican ideas alrededor de esas obras de arte, documentos o restos arqueológicos. (Santos Mateo 2013)

\subsubsection{Entre la historia y la modernidad Casa Museo Remigio Crespo}

En el último proceso de restauración y entrada en funcionamiento, La Casa Museo Remigio Crespo Toral, convertida desde 1947 en Museo de La Ciudad, determinó ingresar a los equipamientos tecnológicos a través del uso de aplicaciones, pantallas digitales y manejo de la plataforma Facebook.

La administración de la Casa Museo concibe a la hipercomunicación en dos direcciones: la primera mediada por plataformas digitales y bondades de conexión a través de la Internet, que caracteriza a este nuevo espacio sociocomunicativo con la posibilidad de interactuar y relacionarse con otros usuarios, conocidos o no, con los que se comparte alguna inquietud, motivación, afición o incluso con los que apenas se comparte nada (Ayala P., 2015). Y la segunda, que es hiper (exceso) conectada de manera directa, cara a cara, esto es en relación con la gente, con el espacio barrial con las visitas de los vecinos al museo y viceversa.

El uso de las herramientas tecnológicas tiene como prioridad en materia de comunicaciones: conectarse con los públicos y llegar a nuevos protagonistas como son los jóvenes, relacionarse con ellos e interactuar. Así mismo, entregar información sobre el museo, los horarios.

La cuenta de Facebook Casa Museo Remigio Crespo reporta más de 4.500 seguidores a las acciones del museo. Los community se encargan de subir la información, crear comunidades, relaciones con establecimientos educativos. Como medio de enlace el Facebook es efectivo permite a los emisores extender respuestas inmediatas a las inquietudes de la gente, la actividad a través de este canal es constante. El museo no dispone de una página independiente, es parte de la web del GAD Municipal, a través de la Dirección Municipal de Cultura y Deportes; una página informativa que enlaza con la cuenta de Facebook. Tampoco cuenta con Twiiter o Instagram, porque la información que se genera en el museo, es manejada por las cuentas de la Dirección de Comunicación del GAD Municipal.

En tecnología intramuseo, la Casa Museo optó por entrar al mundo de la realidad aumentada, a través de la aplicación "aurasma" multiplataforma que dispone de apps para IOS (iphone, Ipad), Android y aplicación web Aurasma Studio. Es nueva, se implementó para el retrato de Elia Liut (Italiano que el 4 de noviembre de 1920, al mando 
del avión El Telégrafo se convirtió en el primer hombre que cruzó los Andes ecuatorianos en un vuelo).

En el costado izquierdo del retrato en óleo de Elia Liut está la cédula que indica como bajarse la aplicación "aurasma", buscar el canal de la Casa Museo Remigio Crespo y suscribirse. Cumplido este proceso, se enfoca la cámara del móvil al retrato de Elia Liut el aparato capta la imagen e inmediato le enlaza con la información que se lo lleva en el celular sobre este personaje. Funciona con plan de datos o wifi. De esa manera el usuario vive la realidad aumentada; es decir,

Esta es una muestra de cómo de a poco, las tecnologías se implementan en el Museo. No es la única APP, hay otra denominada Red Museos Cuenca, creada en el 2015, que posibilita escanear con un código QR, para que el acceso a la información de algunas obras. Esa aplicación es para todos los museos y espacios culturales que están en manos de la Municipalidad de Cuenca.

Parte de la dotación tecnológica en el Casa Museo está marcada por el uso de una pantalla triple para proyección de videos. Una de las salas exhibe esta rectangular pantalla digital donde ruedan los videos que por una parte muestran de manera explicativa la exposición de turno; y por otra los rostros del barrio, del espacio donde se ubica el museo que entra en relación geográfica con otros museos como: Culturas Aborígenes, Manuel Agustín Landívar y el mismo Pumapungo. Esta forma parte de un sistema de comunicación con los públicos y de la parte educativa, funciones fundamentales determinadas por el museo y se relacionan con temas técnicos de muesografía.

\subsubsection{Pumapungo en Internet}

La red es el medio de publicación más barato y de mayor difusión, uno de sus principales atractivos es que, por sus características propias permite que todos los que tienen acceso a un ordenador y una conexión se conviertan en receptores y difusores de la información. (Rivero, 2001)

En el Museo Pumapungo la visión y misión acoplarse a la tecnología empieza en el nuevo milenio entrando al mundo de las plataformas digitales como la web; las redes sociales desde la segunda década del siglo y la implementación de aplicaciones para autoguía. Pumapungo está en la web del Ministerio de Cultura como tal, y en la página propia del Museo donde engloba sus tres ejes: el Museo, Teatro y Parque arqueológico. La fanpage de Facebook es una página multimedia, están desde los perfiles hasta videos, fotografías, textos informativos algunos hipervinculados, "permite colgar información dinámica", dicen Tamara Landívar, directora y Hernán Rodríguez, el comunity.

En Twitter la cuenta @PumapungoMCYP se reactivó hace meses, tiene pocos seguidores, 200; mientras en el Facebook, los seguidores alcanzan los 1990 está rediseñada. Facebook es una plataforma para la generación de contenidos, como también para la interactividad con públicos, investigadores, estudiantes, una interconexión diversa. Como diría Mónica Viñaras (Viñarás Abad, 2012) La función de los museos de difundir la cultura y hacerlo con éxito les obliga a desarrollar una serie de actividades y de darlas a conocer que no tienen de una manera tan directa otras instituciones públicas

El uso de la tecnología, en Pumapungo está concebido desde la guía a través de una aplicación y en torno a eso se crea y está en proceso continuo de mantenerla "Pumapungo APP" diseñada para Smartphone y iPhone, como herramienta de guianza que muy pronto entrará en vigencia dentro de la Sala Arqueológica, se trata de un interactuardor de multimedia que permite guiar a visitantes, mediante un escaneo de código QR, colocado en las vitrinas o maquetas de exhibición.

La aplicación entró a pruebas de ensayo con visitantes, niños de escuelas, que se acercan a la sala, se conectan con plan de datos o wifi, escanean el código QR y empiezan el recorrido. Dos estudiantes de sistemas de la Universidad Politécnica Salesiana Alexis Medina y Paúl Arias desarrollan el sistema, gracias a un convenio de cooperación entre la Universidad, el Museo y la Cátedra UNESCO.

"Pumapungo APP" posibilita a los usuarios seguir las Huellas de la cultura ancestral, que es el bloque donde se muestra y explica la presencia de las culturas precolombinas de esta zona austral ecuatoriana; así mismo lleva a conocer Pumapungo en el tiempo, el bloque central de la sala, y Proyecto Pumapungo, ubicado en el bloque izquierdo de esa sala, donde muestra ese trabajo realizado por historiadores, arqueólogos, antropólogos para conformar lo que hoy es el gran museo.

La información que se obtiene del escaneo al código QR puede archivarse en el móvil y el usuario lleva videos, texto, fotografías de la reserva. El objetivo es que la aplicación englobe la sala etnográfica y otras áreas del gran complejo. Esta es una intromisión en el mundo de las tecnologías y el museo, Algo así como ocurrió en el 2010 con el Streetmuseum, de Londres, la primera app mundial para museos con tecnología de Realidad Aumentada.

Facebook es la reina de las plataformas para museos manejados por entidades estatales y de gobiernos locales, y sin dejar de lado la investigación de Mónica Viñaras. Las posibilidades de participación y colaboración ofrecidas por la tecnología de la Web 2.0 constituyen, como definía el profesor Sáez Vacas (Vacas, 2006) «un nuevo entorno tecnosocial, más que una nueva versión de Internet».

\subsection{Las audioguías parte de la tecnología}

Ahora los recorridos pueden ser de manera virtual o física, y en el caso del Museo de la Catedral Vieja , museo de arte religioso, las guías son a través de equipos de audio. Cuando el visitante llega y decide recorrer el museo de arte religioso, pide un aparato Audio Conexus Inc, de tecnología canadiense propia para museos, se programa en el idioma que desee, puede ser español, inglés o francés, y con hacer play, el recorrido por las diferentes obras y espacios empieza. 
La implementación tecnológica de guías con audífonos se instaló en mayo de 2017, con el objetivo de ampliar el servicio con otros idiomas, lograr mayor versatilidad y mejor servicio a los públicos. Esta es la tecnología de mayor connotación que dispone el museo, un story telling grabado en el dispositivo, con 20 pistas que recogen el contenido de toda la muestra, explica de manera oral los diferentes espacios de la Catedral Vieja, antigua Iglesia de El Sagrario. Cada pista sigue un guion narrativo, relato hecho por el equipo de la Catedral.

Y en materia de plataformas digitales, este museo le apuesta la divulgación y producción de contenidos para Facebook, un canal de interactividad e interconectividad con los públicos, y donde la imagen es el punto de atracción; fotografías, algunos álbumes con información para la promoción de los eventos culturales ya sean conciertos de las Orquestas Sinfónicas o conversatorios. Las fotografías del perfil se renuevan cada seis meses.

El uso de la red social tiene un objetivo: mejorar la relación, conexión y contacto con los públicos. La plataforma digital permite crear grupos o comunidades virtuales, son colectivos que se generan y se enlazan a las acciones culturales que hace la Catedral Vieja. El manejo de públicos extranjeros para los conciertos de las sinfónicas se hace a través de "Gringo post", (grupo de voluntarios con el deseo común de ofrecer anuncios clasificados gratuitos e información a la comunidad de expatriados) mientras el equipo de la Catedral trabaja con públicos locales, difunden mensajes para ellos con el fin de informar a la ciudadanía lo que en el espacio puede encontrar.

La necesidad de manejar estos canales es cada vez mayor por la aproximación y enlaces que logran con artistas plásticos, músicos, instituciones particulares, que al mirar la página de Facebook reaccionan de dos maneras; visitan la Catedral o se conectan por medio de la computadora.

\subsection{Los Espacios culturales y su uso de las plataformas digitales}

\subsubsection{La Mata del Frío Asesino}

Las tecnologías son las mismas, lo que se mide en el uso de ellas es la estrategia que cada museo, y no solo ellos, sino los espacios culturales que están inmersos en esta actividad, utilicen para proyectarse a través de la red.

Un grupo de jóvenes artistas cuencanos pasaron por una experiencia que los llevó de la tecnología a físico. Lo que nació como un blog, como una bitácora digital donde se podía hablar, compartir, publicar, etiquetar cosas de arte urbano y de arte en diferentes expresiones, poco a poco se salió de la plataforma digital para convertirse en un espacio donde el contacto ya no era mediado por una computadora, sino frente a frente a frente, "face to face" como lo llaman las generaciones de hoy.

Cualquiera diría que son nuevos y no es verdad, tienen diez años de trabajar en el arte y siempre reciben a nuevos integrantes, esto es seguidores en sus páginas y a gente que quiera unirse para hacer un espacio de expansión artística.
Los jóvenes activistas bautizaron a este espacio como: "La mata del frío asesino", lugar de acción cultural que se ubica en una casona de antaño que de a poco se transforma, porque la restauran respetando su arquitectura original.

"La mata" como la llaman, no solo se quedó en el blog, ahora -y como la mayor parte de la sociedad- se relaciona con los públicos de manera presencial y virtual. Esta segunda interrelación se logra a través de las cuentas de Instagram con 800 fans, Facebook con 2000 seguidores y Twitter que está dormido.

Actualizar los contenidos, reaccionar a los like, a los comentarios, brinda a los emprendedores la posibilidad de tener una idea de su trabajo y su proyección fuera de las paredes de la casa patrimonial. Para "La mata" lo más bondadoso de esta interacción virtual, en la que ya están cinco años, es el acercamiento del espacio cultural a los públicos y viceversa. La visión de Joaquín Pérez, el impulsor y sus amigos es que la imagen esa "mata" llegue y se proyecte a Manhattan, a París, a México; a lugares grandes estratégicos para el arte y la cultura, a través de las plataformas en red.

El ingreso al nuevo milenio no fue sólo una transformación de número. El inicio del nuevo milenio fue una época de transformación social universal y comunicacional dictadas por los avances tecnológicos. Es la era de lo digital, arrinconando a lo convencional tradicional. En la computadora está la posibilidad de conexión con las páginas de YouTube, Facebook, Instagram donde se encuentran videos colgados incluso hace tiempos, pueden ser décadas y cada vez suman seguidores, visitas, comentarios.

Hoy todo es digital, desde los aparatos eléctricos de casa, hasta las relaciones interpersonales virtuales marcadas por un aparato que se dice digital. Se habla de la "biblioteca digital", del "arte digital", de la "universidad virtual", del "museo virtual" para referirse a estos ámbitos, como a tantos otros, afectados por las TICs. (Ristol, 2015)

El manejo de los soportes digitales para la creación artística está ligado, muchas veces a la acción ya sea de museos y espacios o centros culturales, la Internet se ha convertido en la herramienta de intercambio de obras y saberes, incluso hoy los mercados del arte son por Internet.

\subsubsection{Yuyay Arte en manos de un artista en red}

Las experiencias sobre este contacto vía red en Cuenca tienen una voz, Diego Muñoz quien en el 2016 instaló Yuyay Arte, un espacio de acción cultural, enfocado hacia la pintura y con énfasis en los públicos infantiles ya sea para los talleres continuos durante todo el año y para las exposiciones.

En el Facebook, Yuyay Arte tiene cerca de 600 seguidores. Esta plataforma multimedia presenta fotografías, anuncios de la Casa del Pintor. El "face" es un canal de promoción del espacio cultural que es un centro de varias acciones. Una cuenta de twitter, no muy voluminosa, cuenta con 57 seguidores, un http://cuencadiegomunoz.blogspot.com/, creado en el 
2010, mantienen conectado a la producción de Diego con el mundo, un artista interconectado, que está en la red gracias a las bondades de la Tics.

La exploración a la tecnología por parte de Diego empezó en el 2002 con los videos celulares, obras con las que ganó un premio en México. Un "largo-corto-metraje" hecho en celular, cosa aparentemente rara pero no imposible, medio de no creer dentro del mundo de las expresiones de inicio de milenio.

La página web y demás plataformas son una puerta de entrada a los múltiples servicios y ofertas, y la conexión a otros espacios digitales indispensables dentro de la interacción con los públicos: las cuentas de redes sociales. Twitter, Facebook son útiles para etiquetas información, mientras Instagram lleva cierto favoritismo para compartir álbumes de fotos.

Marcela Ristol (2015), investigadora argentina sobre arte y museos toma una cita de Bellido Gant (s.f)170 que, dice "la tecnología ha potenciado la función difusora de los museos y a su vez ha permitido desarrollar dos nuevas tipologías: los museos virtuales y museos y galerías digitales vinculados a la cibercreación”. Una primera forma de digitar el museo sin duda es encontrar una página web institucional y la posibilidad de entrar en ella como si se ingresara por la puerta misma del museo.

\subsection{De lo convencional a lo digital El Prohibido Centro Cultural}

La diferencia generacional en materia de tecnologías e Internet se mide desde dos etapas: generaciones que migraron hacia el mundo de lo digital y los nativos digitales. Los integrantes del primer grupo no escapan a la interconexión y los artistas, gestores que surgieron en las últimas décadas del siglo y milenio pasado hoy están en red, atravesando algunas fronteras como es el caso de El Prohibido Centro Cultural.

De los postes a las redes sociales, pasando por experimentar una wordpress. Como artista plástico y como gestor cultural, Eduardo Moscoso nunca dudó en entrar al uso de las tecnologías de la comunicación e información para no solo promocionar El Prohibido como espacio para la promoción cultural alternativa, sino para entrarle al mundo de las comunidades virtuales con gente que compartía su mismo afán, sus mismas curiosidades.

El no tan famoso, pero casi olvidado "hi5" fue una de las primeras formas de interconectarse a la que apostó Moscoso, por una razón: el espacio en los medios masivos a propuestas de arte alternativo era nulo, no estaba avalado por el oficialismo; y claro, las redes salieron y con ellas se armaron sus propias páginas, canales informativos que restaron y restan importancia a los medios convencionales, porque dejan mayor posibilidad de interconexión con públicos y actores.

Las plataformas digitales abrieron la ventana para visibilizarse no solo de manera local sino mundial y para crear agendas de información con la programación planificada.
Todo lo que la tecnología ofrecía, El Prohibido lo adoptaba. Página web, que no resultó ser muy efectiva; blog en wordpress y nada mejor que las redes sociales, cuenta en Facebook, con más de 8.000 seguidores, Instagram, Twitter a través de la cuenta personal de su dueño Eduardo Moscoso con 500 seguidores, espacios abiertos a la dinámica comunicativa, de información y enlaces.

Hay eventos que llegan a tener hasta 12.000 me gusta, y los seguidores de El Prohibido en el "face" supera esa cifra. Comprar publicidad en esta red es una estrategia de mercado que Moscoso la ve con buenos resultados, es barato y tiene muchos alcances. EL Prohibido es uno de lugares que visita el turista, son gente de afuera que se acerca al espacio y muchos mantienen contacto primero con las páginas digitales.

Aplicadas al entorno de los museos y de los espacios de acción cultural, las tecnologías 2.0 sirvieron para que la participación de los visitantes se incorporase a tres espacios diferenciados. En primer lugar, dentro de la propia web del museo, principalmente a través de blogs, segundo lugar, en repositorios externos donde compartir materiales audiovisuales: vídeos en You Tube, fotos en Flickr, audios en iTunes o presentaciones en SlideShare. Y, en tercer lugar, en las redes sociales, principalmente a través de Facebook y Twitter. (del Río Castro, 2011)

\subsection{Break Espacio de Arte y Cultura}

En los últimos tiempos Cuyker despunta como la red que conecta con la agenda de eventos culturales, sociales, deportivo, académicos científicos. Los jóvenes, nativos digitales, son quienes en mayor grado acceden a ellos, y en esta onda quienes hacen gestión cultural desde la iniciativa privada le apuestan a anunciarse en esta plataforma Ese es el caso de Break que anuncia la programación para teatro, música, música en livingroom, la especialidad de este centro. El Cuyker es una red social a la que se puede acceder incluso a través del Facebook, detalla la agenda cultural de la ciudad y se la puede descargar al móvil como una aplicación.

La red da cuenta del número de visitas que tiene el anuncio, además de texto, un mapa, es un canal de información y contacto, es parte del mundo digitalizado en el que se desenvuelven porque toda la información se carga en un teléfono móvil. Para lanzar un evento en Cuyker hay que ser estrategas en el manejo del tiempo, la imagen; especificar, promociones, si es invitación personalizada. Texto y foto comandan en esta red social.

Los Emprendedores en materia de cultura no miran, ni planifican sus proyectos sino es con difusión de imagen y accionar en plataformas digitales, y dándoles a cada una de ellas estrategias. Todas permiten conectividad e interactividad, básico en el accionar cultural de hoy. Los expertos en redes, argumentan que Cuyker es una red social preferida por extranjeros, en Cuenca; Facebook aglutina a todas las edades, por eso la cuenta de Break tiene180 seguidores e Instagram está recién abierto. 
Información, promoción, enlaces, contactos, lo que más ayuda en la proyección de imagen desde lo virtual son los estados, ya sea de Facebook, de Instagram. Esos estados son un empujón para actualizar la información, de videos donde se presenten como en pastillas, el estilo del grupo en música, la obra a desarrollar en teatro, animando al público a participar de los actos de arte.

\section{Concluciones}

La actividad comunicacional y de promoción de algunos museos y centros culturales de la ciudad está mediada por las plataformas digitales. En principio fue la web, luego las redes sociales que mantienen un alto nivel de aceptación y de acción. Esas son plataformas que posibilitan no solo la interactividad y multimedialidad, sino la posibilidad de generar discursos hipermediales, esto es manejar hipervínculos para que los usuarios naveguen de acuerdo a sus intereses.

Las páginas web no son plataformas para le emisión de discursos informativos, sino para discursos relacionados con la filosofía y razón de ser de las corporaciones. La acción y gestión de los museos y centros culturales en materia de comunicación, marketing y promoción están en las redes sociales, cada uno de ellos tienen en promedio de tres a cinco publicaciones diarias.

Los usos de la tecnología dentro del accionar comunicacional y de atención a los públicos de los museos no están marcados solo por el uso de las plataformas 2.0, sino por los equipos y plataformas intra-museos esto es la creación de aplicaciones para equipos móviles que, por una parte, median en la guianza interna; pero también además posibilita llevar información al usuario en su dispositivo.

\section{Recomendaciones}

Elevar el nivel de interacción con los públicos a través de la creación de comunidades virtuales integradas por actores, gestores, académicos, críticos, líderes políticos -sociales. Las comunidades virtuales permiten interactuar e intercambiar iniciativas que eleven el quehacer museístico y cultural a un proceso de desarrollo económico y ampliar la cobertura para una mayor recepción y acción.

La actualización de los contenidos es una de las características en el manejo de la difusión por las plataformas digitales. Las divulgaciones a través de las redes sociales tienen que manejar el tiempo real, el tiempo sin tiempo, es decir la información no pierde vigencia, y el tiempo de proyección al futuro.
La implementación intramuseo es una de las estrategias de comunicación que no puede obviarse en los espacios cultural. Poseer una aplicación es llevar la información en todo momento, y los museos como los centros son lugares donde se genera no solo eventos, sino corrientes académicas, científicas y de investigación.

\section{Referencias Bibliográficas}

Acha Barral, M. d. R. (2006). Dialéctica y nuevas tecnologías de la información en los. En La tecnología y la comunicación museística (p. 44). Murcia: Comunidad Autónoma de la Región de Murcia.

Ayala P., T. (2015). Redes sociales, poder y participación ciudadana. Revista Austral de Ciencias Sociales, 31.

Castells, M. (1997). La Era de la Información tomo I: Economía, Sociedad y Cultura. Madrid.

Crespo, J. L. (2012). Estudios sobre Arte y Comunicación Social. Colección CABA, Cuadernos Artesanos de Bellas Artes.

Fiallos Quinteros, B. (2015). E-COMUNICACIóN Y CONTENIDOS DIGITALES EN LOS MUSEOS DE ARTE DEL SIGLO XXI. Tsantsa. Revista de Investigación Artística, 5.

Martínez-Sanz, R. (2012). Estrategia Comunicativa Digital en el Museo. Descargado de http://eprints .rclis.org/17311/1/391-395.pdf

Ristol, M. (2015). Museos y gestión del arte. Sevilla: Universidad De Olavide - España.

Rivero, P. (2001). Museo e Internet. Zaragoza, España.

Vacas, F. R. A. \&. S. (2006). Blogs: en la vanguardia de la nueva generación web. novática. Archivo Digital $U P M, 1$.

Viñarás Abad, M. (2012). Claves para la participación y. Madrid Universidad San Pablo CEU, España.

Recibido: 30 de junio de 2018

Aceptado: 3 de septiembre de 2018 
